\title{
Intraflagellar Protein 88 Interactome Analysis: A Bioinformatics Approach Highlights Its Role in Testis and Sperm Function
}

\author{
Maria João Freitas, Margarida Fardilha \\ Signal Transduction Laboratory, Centre for Cell Biology, Health Sciences Department and Biology Department, \\ University of Aveiro, Aveiro, Portugal \\ Email: mfardilha@ua.pt
}

Received 9 April 2014; revised 8 May 2014; accepted 9 June 2014

Copyright (C 2014 by authors and Scientific Research Publishing Inc.

This work is licensed under the Creative Commons Attribution International License (CC BY).

http://creativecommons.org/licenses/by/4.0/

(c) (i) Open Access

\begin{abstract}
Intraflagellar transport (IFT) is essential for cilium and flagellar assembly. This movement is accomplished by two IFT complexes: A and B. IFT88, intraflagellar protein 88, is a core element of IFT complex B. This protein has been linked to migration, to olfactory function, spindle formation and to mitosis. Recently, IFT88 was identified as a TCTEX1D4 interacting protein in human testis, suggesting a role in male reproduction. To broaden the knowledge on IFT88 function, particularly in testis and spermatozoa, an in silico analysis of IFT88 and IFT88 interactome was undertaken. IFT88 appears to be prone to protein-protein interactions, involved in spermatogenesis and since it interacts with key proteins related to male fertility, it may have a role in reproduction.
\end{abstract}

\section{Keywords}

Intraflagellar Protein 88 Homolog, Spermatozoa, Network

\section{Introduction}

Intraflagellar transport (IFT) was described for the first time, in Chlamydomonas reinhardtii flagella, twenty years ago [1]. It is characterized by the bi-directional movement of cargo along the flagellum or cilium and is independent from the flagellar betting and essential for cilium and flagellar assembly [2] [3]. There are three essential components for the intraflagellar transport: 1) the microtubules provides the physical support for the movement; 2) the molecular motors, kinesin and dynein power the movement and 3) the IFT complexes mediate interactions between molecular motors and cellular cargos [4]. 
The IFT complexes (A and B) are composed of combinations of at least 20 different IFT proteins [2]. Each IFT protein is essential for cilium assembly and function. The importance of these proteins in cellular functions is highlighted by ciliopathies, such as retinal degeneration and defective determination of the left-right axis [5]. The IFT-A complex appears to be essential for the retrograde transport [6] while IFT-B complex is the core of the IFT transport [2]. Besides the role in IFT transport, IFT proteins are crucial in ciliogenesis [7] and mitosis [8].

Intraflagellar protein 88 (IFT88 or Tg737) is a core protein of the IFT-B complex [2]. It was first described associated with an autosomal recessive form of polycystic kidney disease [9]. The presence of some IFT88 polymorphisms enhances the probability of cystic renal diseases [10]. Recently, IFT88 has also been linked to migration and invasion of hepatoma cells [11], to olfactory function [12], spindle formation [8], and to mitosis (a non-cilium function) [13]. Recently a yeast two hybrid screen performed in our laboratory revealed that, in testis, IFT88 interacts with TCTEX1D4 (t-complex testis expressed 1 domain containing 4), a dynein light chain from the DYNT1/TCTEX1 family [14]. TCTEX1D4 was first described as an interactor of several components of the TGFbeta pathway [15]. Yet Fardilha et al. identified TCTEX1D4 as a phosphoprotein phosphatase 1 interactor in human testis and Korrodi-Gregório showed that TCTEX1D4 is involved in microtubule organization. Thus, the data points to a role of IFT88 in spermatozoa, particularly in the flagellum.

Here propose an in silico approach to unravel putative functions for IFT88, particularly in testis and spermatozoa, and therefore understand its role in cell physiology.

\section{Material and Methods}

\subsection{IFT88 in Sillico Analysis}

IFT 88 aminoacid sequence was retrieve from UniProt database and used for further protein sequence analysis. For secondary structure Eukaryotic Linear Motif (ELM) [16] and Psipredv3.3 (http://bioinf.cs.ucl.ac.uk/psipred/) were used. INTERPRO, ScanProsite [17] and SMART [18] (26. 2. 2014) analyzed the IFT88 sequence and determine possible structural domains (only scores with e-value smaller than 0.01, high confidence score and list in two of the three databases were considered). TPRpred was used to profile the representation of tetratrico peptide repeats (only scores with p-values lower that 0.01 were considered) [19].

Several bioinformatics tools inferred the putative phosphorylation sites (02.02.2014): Diphos 1.3

(http://www.dabi.temple.edu/disphos/ ), NetPhosK [20], NetPhos 2.0 [21] and ELM. Other putative post-modification, such glycosylation were searched through Prosite [22]; ELM; NetNGlyc 1.0

(http://www.cbs.dtu.dk/services/NetNGlyc/), SignalP 1.4 [23]; Myristoylator [24] and NMT

(http://mendel.imp.ac.at/myristate/SUPLpredictor.html). Only results with scores above 0.7 and/or with high conservations scores were considered. Aminoacid absolute surface accessibility was analyzed by NetSurfP 1 [25]. PPP1 binding motifs were search using ScanProsite (3.2.2014).

\subsection{IFT88 Interactome Analysis}

IFT88 interactome was retrieved from several databases using the Psiquic View tool (30.1.2014) [26]. Thirtyeight interactors were obtained. IFT88 network was designed using Cytoscape 3.0.2. The interactions of each IFT88 interactor was retrieved using PSICQUICU niversal Client plugin (28.02.2014-IntAct, MINT and STRING databases) and only interactions between IFT88 interactors were considered. Expression of all IFT88 interactors plus protamine 2, a testis specific protein, was obtained from UNIGENE. Testis expression level of all TCTEX1D4 interacting proteins was converted to \% of transcripts in testis. TissueNet database was used to recover testis expression of IFT88 interactors (both RNA and proteins levels) and putative identify testis specific interactions of IFT88 (25.2.2014). Phenotypes associated with IFT88 interactors were retrieved from Mouse Genome Informatics (MGI) (http://www.informatics.jax.org/) using the Uniprot ID and from Phenopedia using the keyword "male infertility" (4.2.2014). Biologic Process, Molecular Function and Cellular Component classification was obtained using the ClueGo v1.7.1. Only results with statistic significance were considered (p value corrected with Bonferroni < 0.01) and only experimental inferred data.

\section{Results}

\subsection{In Silico Characterization of IFT88}

A bioinformatic analysis of IFT88 was performed for the first time. The goal was to identify physiological rele- 
vant motifs and post-translational modifications for testis and spermatozoa function. Figure 1 shows that IFT88 is mainly a globular protein, according to ELM and Psipred. However the N-terminus and C-terminus are disordered (1 - 138 and 738 - 833). Curiously, the globular domain is formed only by alfa helixes. Regarding putative motifs in IFT88, two tetratricopeptide repeat-containing (TRP) domains were identified. The first domain is composed of three TRP repeats of TRP motifs while the second domain consists o 9 TPR motifs. ITF88 may be phosphorylated at the S612 and T497. Although S612 and T497 are localized in the globular domain-which makes them theoretically less accessible to phosphorylation-serine 612 presents an absolute surface accessibility of 0.72 , which indicates a strong possibility of phosphorylation. According to NetPhosK, Ataxia telangiectasia mutated kinase is the proposed kinase to phosphorylate S612 and Protein kinase C is putatively responsible for T497 phosphorylation. No potential tyrosines phosphorylation sites were found. No other post-translational modifications were identified in IFT88. Two Phospho Protein Phosphatase 1 binding motifs were identified corresponding to a SILK (242 - 245) and a RVxF (260 - 264) motif [27].

\subsection{IFT88 Interactome Analysis}

The IFT88 interactome was retrieve from several databases, using the Psiquic View tool. Table 1 lists the thirty eight identified interactors for IFT88, the protein-protein interaction (PPI) identification method and the Uniprot ID of each interactor. 76\% of interactions were identified using indirect approaches (such as text mining and interlog mapping) while $24 \%$ were detected by direct methods (yeast two hybrid system and biochemical methods). It is noteworthy to mention that $21 \%$ of IFT88 interactors are members of the IFT family.

Figure 2 is a graphical representation of IFT88 network. It shows the direct interactions between all IFT88

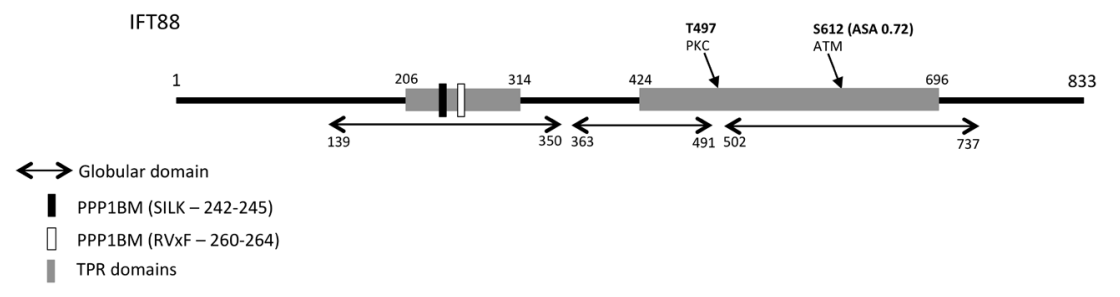

Figure 1. Schematic representation of human IFT88. IFT88 presents three globular domains as well two TPR doamins. Putative phosphorylation sites and PPP1BM are also represented. ASA, absolute surface acessibility; ATM, ataxia telangiectasia mutated; PKB, protein kinase $\mathrm{B}$; TPR, tetratricopeptide repeat; PPP1BM, PPP1 binding motif.

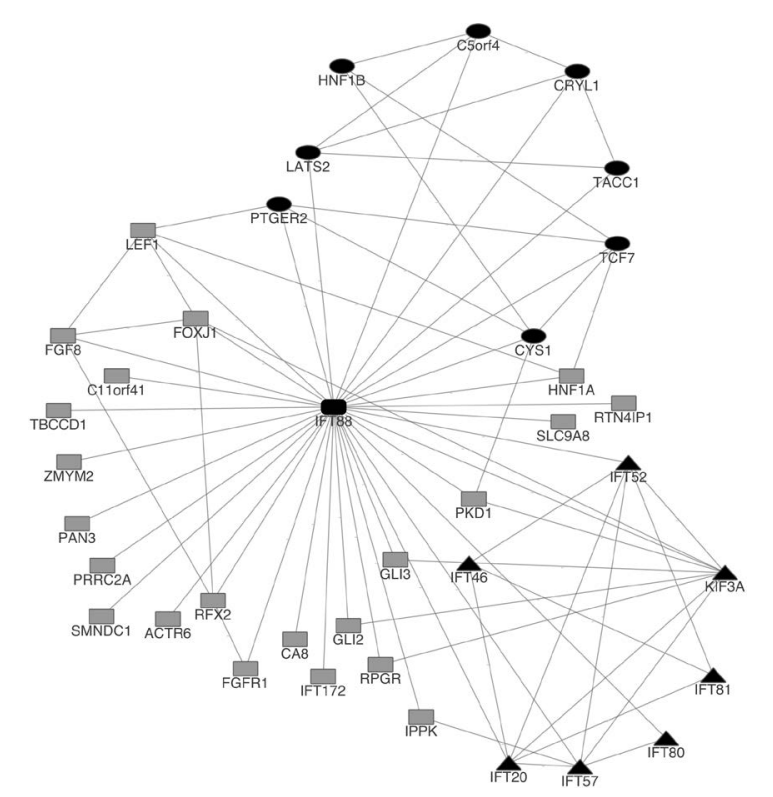

Figure 2. IFT88 network. The network was constructed using the software Cytoscape 3.0.2. 
Table 1. Partial human IFT88 interactome. Interactors were retrieved from Psiquic View using the plugin PSICQUIC Universal Client from Cytoscape 3.0.2.

\begin{tabular}{|c|c|c|c|}
\hline Interactors & Synonyms & PPI detection method & UNIPROT ID \\
\hline Actin-related protein 6 & ACTR6 & interologs mapping & Q9GZN1 \\
\hline Carbonic anhydrase-related protein & CA8 & predictive text mining & Q32MY2 \\
\hline Chromosome 5 open reading frame 4, isoform CRA_a & C5orf4 & predictive text mining & Q96IV6 \\
\hline Cystin-1 & CYS1 & predictive text mining & Q717R9 \\
\hline DNA-binding protein RFX2 & RFX2 & predictive text mining & Q8SNA2 \\
\hline Fibroblast growth factor 8 & FGF8 & predictive text mining & P55075 \\
\hline Fibroblast growth factor receptor 1 & FGFR1 & predictive text mining & P11362 \\
\hline Forkhead box protein J1 & FOXJ1 & predictive text mining & Q92949 \\
\hline KIAA1549L or C11orf41 & & two hybrid & Q6ZVL6 \\
\hline Hepatocyte nuclear factor 1-alpha & HNF1A & predictive text mining & P20823 \\
\hline Inositol-pentakisphosphate 2-kinase & IPPK & predictive text mining & Q9H8X2 \\
\hline Intraflagellar transport protein 172 homology & IFT172 & predictive text mining interologs mapping & Q9ULP1 \\
\hline Intraflagellar transport protein 20 homolog & IFT20 & predictive text mining & Q8IY31 \\
\hline Intraflagellar transport protein 46 homolog & IFT46 & biochemical & Q9NQC8 \\
\hline Intraflagellar transport protein 52 homolog & IFT52 & interologs mapping & Q9Y366 \\
\hline Intraflagellar transport protein 57 homolog & IFT57 & predictive text mining & Q9NWB7 \\
\hline Intraflagellar transport protein 80 homolog & IFT80 & interologs mapping & Q9P2H3 \\
\hline Intraflagellar transport protein 81 homolog & IFT81 & biochemical & Q8WYA0 \\
\hline Intraflagellar transport portein 88 homolog & IFT88 & affinity chromatography technology & Q8N719 \\
\hline Kinesin-like protein KIF3A & KIF3A & predictive text mining interologs mapping & Q9Y496 \\
\hline Lambda-crystallin homolog & CRYL1 & predictive text mining & Q9Y2S2 \\
\hline Lymphoid enhancer-binding factor 1 & LEF1 & predictive text mining & Q9UJU2 \\
\hline PAB-dependent poly(A)-specific ribonuclease subunit 3 & PAN3 & interologs mapping & Q58A45 \\
\hline Polycystin-1 & PDK1 & predictive text mining interologs mapping & P98161 \\
\hline Proline-rich and coiled-coil-containing protein 2 & PRRC2A & coimmunoprecipitation two hybrid & P48634 \\
\hline Prostaglandin E2 receptor EP2 subtype & PTGER2 & predictive text mining & P43116 \\
\hline Reticulon-4-interacting protein 1, mitochondrial & RTN4IP1 & interologs mapping & Q8WWV3 \\
\hline Serine/threonine-protein kinase LATS2 & LATS2 & predictive text mining & Q9P2X1 \\
\hline Sodium/hydrogen exchanger 8 & SLC9A8 & interologs mapping & Q9Y2E8 \\
\hline Survival of motor neuron-related-splicing factor 30 & SMNDC1 & anti bait coimmunoprecipitation two hybrid & O75940 \\
\hline TBCC domain-containing protein 1 & TBCCD1 & $\begin{array}{c}\text { fluorescence microscopy } \\
\text { experimental interaction detection }\end{array}$ & Q9NVR7 \\
\hline Hepatocyte nuclear factor 1-beta & HNF1B & predictive text mining & P35680 \\
\hline Transcription factor 7 & TCF7 & predictive text mining & Q9UKI4 \\
\hline Transcriptional activator GLI3 & GLI3) & experimental knowledge based & P10071 \\
\hline Transforming acidic coiled-coil-containing protein 1 & TACC1 & predictive text mining & Q9UPP9 \\
\hline t-complex testis expressed 1 domain containing 4 & TCTEX1D4 & Two hybrid & Q5JR98 \\
\hline X-linked retinitis pigmentosa GTPase regulator & RPGR & experimental interaction detection two hybrid & Q92834 \\
\hline Zinc finger MYM-type protein 2 & ZMYM2 & predictive text mining & Q9UBW7 \\
\hline Zinc finger protein GLI2 & GLI2 & experimental knowledge based & P10070 \\
\hline
\end{tabular}


interactors. As it is possible to observe there are two groups in the network. The first is characterized by the interconnectivity between all IFT proteins, creating a subnetwork (triangle shape nodes). Also, in this group, kinesin-like protein 3A (KIF3A) interacts with four IFT protein. LATS2, HNF1B, C5orf4, CRYL1, TACC1, TCF7, PTGER2 and CYS1 compose the second group (circle shape nodes). Between these proteins there is high connectivity (one protein interacts with at least two other).

Testis expression of all IFT88 interactors plus protamine 2 (PRM2) was obtained. Protamine 2 is a testis-specific protein responsible for highly packing the spermatozoa DNA [28]. Since the purpose of the expression profile was to identify testis-specific proteins, PRM2 was used as a control. None of IFT88 interactors presents a profile similar to PRM2 and therefore none of the interactors is a testis-specific protein. Except for RFX2, which has a testis expression of 23\%. All IFT88 interactors have less than 20\% testis expression. To specify IFT88 interactions that can occur in testis, Tissue Net was used. Two IFT88 interactors were identified as occurring in human testis: PRRC2A and SMNDC1 (at RNA level), together with IFT88. With the purpose of further identify IFT88 interactors that have a crucial role in male fertility, a search in Mouse Genome Informatics and Phenopedia databases was performed. FGF8, FOXJ1, HNF1A, PDK1, PTGER2, SLC9A8, GLI3 and GLI2 when knock out in mouse present phenotypes that interfere with spermatogenesis, sperm cells physiology, histology and morphology of male reproductive system and therefore can be associated with male infertility. IFT88 network was characterized regarding Biological Process, Cellular Component and Molecular Function. Table 2 shows that only six proteins were classified and none in the molecular function category. Regarding Biological process urogenital system development is the most represented process. However, it is interesting that three proteins, FGF8, HNF1B and PDK1 are associated with reproduction, more specifically in sex differentiation. Furthermore, the only cellular component represented is the cilium, in which GLI3 and RPGR are putatively located.

Table 2. Biological process and cellular component of IFT88 interactors. The data was retrieved using the plugin ClueGo v1.7.1 from Cytoscape 3.0.2.

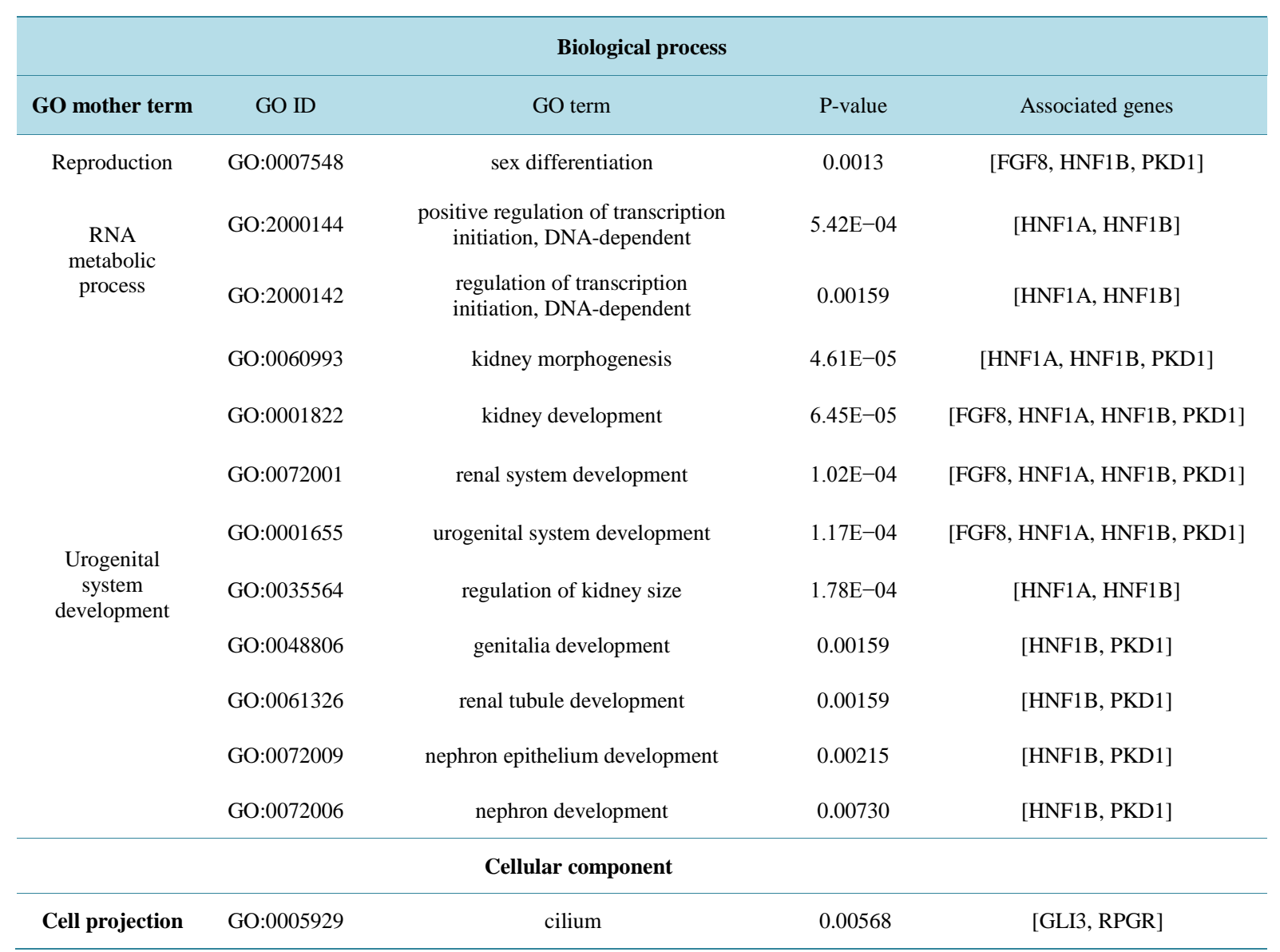




\section{Discussion}

IFT88 was first identified in the Chlamydomonas reinhardtii flagella [1]. Since then, IFT88 has been implicated in several processes, such as mitosis and migration. Nevertheless there is a lack of information regarding IFT88 function. To fill this gap, an in silico analysis of IFT88 structure and network was undertaken.

Two putative TP domains were identified in IFT88. TPR domains are composed of a degenerate 34-amino acid repeat motif that usually appears arrayed [29]. These domains are conserved throughout evolution, being identified in bacteria and humans. TPR are widespread in a high range of proteins and appear to be involved in distinct processes such as cell-cycle control, transcription and protein kinase regulation. The presence of TPR domains is correlated with protein-protein interactions (TPR-TPR interactions or TPR-nonTPR interactions) and assembly of multiprotein complexes [30]. The fact that IFT88 presents two domains hints a role in protein- protein interactions, which is not surprising, since it is a central protein in the IFT complex B. However, only IFT172 presents also TPR domains. This may indicate that TPR domains are not necessary for the IFT complex B assembly but for binding of cargo to the IFT complex.

Two predicted phosphorylation sites may occur in IFT88, one is the S612 and the other in T497. However, theoretically, S612 is more prone to phosphorylation since it is more accessible. Serine 612 is putatively phosphorylated by protein telangiectasia mutated kinase (ATM). The knockout of ATM produces an infertility phenotype, characterized by impaired spermatogenesis [31]. The fact that IFT88 is putatively phosphorylated by ATM and has been described as crucial for mitosis [8] may hint a role in spermatogenesis.

An RVxF and SILK motifs, were identified in IFT88. RVxF motif is the best characterized PPP1BM. Most PPP1 interacting proteins present a RVxF. Curiously, the binding of RVxF does not affect the conformation and activity of PPP1 but promotes other interactions [32]. SILK motifs are normally N-terminal to RVxF motifs and influence the binding of other motifs, namely RVxF. The fact that IFT 88 presents both PPP1BM is a good hint for the possibility of being a PPP1 interactor protein.

IFT88 interactome was retrieve from several databases and TCTEX1D4 was added to the interactome since IFT88 was identified as a TCTEX1D4 interactor [14]. Most interactors were identified by indirect methods; therefore these interactions must be confirmed by direct methods. The fact that a quarter of IFT88 interactors are other IFT proteins and the interconnectivity between these proteins supports that IFT88 is part of an IFT complex. All IFT proteins identified are part of IFT complex B [2], which strongly suggests that IFT88 is also present in IFT complex B (as described in the literature). Kinesin-like protein 3A (KIF3A) interacts with four IFT proteins. KIF3A, together with KIF3B forms a complex which is capable of anterograde transport of organelles through microtubules [33]. The interaction between IFT proteins and KIF3A suggests that this kinesin can transport the IFT complex through spermatozoa flagellum. A second subnetwork is identified in IFT88 interactome. The proteins that compose this subnetwork are involved, according to the literature, in cancer, renal disease and diabetes [34]-[42]. The interconnectivity between this proteins and the fact that IFT88 interacts with all of them may indicate the involvement of IFT88 in a common pathophysiological mechanism.

To give a biological meaning to IFT88 interactors in testis, testis expression, phenotypes associated, biological process, cellular localization and molecular function were analysed with three IFT88 interactors interesting results were obtained. FGF8 and PDK1 when knock out produce a infertility phenotype as well as appear to be involved in sex differentiation. On the other hand, GLI3, when knockout, also produces a infertility phenotype and appears to be localized in the flagellum. The fact that IFT88 interacts with these may indicate a role in male fertility.

\section{Conclusion}

IFT88 sequence and interactome in silico analysis were performed with the goal of identifying biological meaningful interactions in male reproductive functions. IFT88 is prone to protein-protein interactions since it presents TPR domains and is a part of the IFT complex B. Also, it is putative phosphorylated by ATM and presents two PPP1BM, being thus regulated by two proteins involved in spermatogenesis. The in silico analysis of IFT88 interactome allowed to infer that IFT88 may have a crucial role to male reproduction (FGF8, PDK1, GLI3). It is noteworthy to mention that many more interactors are still to identify, probably some with crucial roles in male fertility.

\section{References}

[1] Kozminski, K.G., Johnson, K.A., Forscher, P. and Rosenbaum, J.L. (1993) A Motility in the Eukaryotic Flagellum 
Unrelated to Flagellar Beating. Proceedings of the National Academy of Sciences of the United States of America, 90, 5519-5523. http://dx.doi.org/10.1073/pnas.90.12.5519

[2] Hao, L. and Scholey, J.M. (2009) Intraflagellar Transport at a Glance. Journal of Cell Science, 122, 889-892. http://dx.doi.org/10.1242/jcs.023861

[3] Scholey, J.M. and Anderson, K.V. (2006) Intraflagellar Transport and Cilium-Based Signaling. Cell, 125, $439-442$. http://dx.doi.org/10.1016/j.cell.2006.04.013

[4] Bhogaraju, S., Engel, B.D. and Lorentzen, E. (2013) Intraflagellar Transport Complex Structure and Cargo Interactions. Cilia, 2, 10. http://dx.doi.org/10.1186/2046-2530-2-10

[5] Badano, J.L., Mitsuma, N., Beales, P.L. and Katsanis, N. (2006) The Ciliopathies: An Emerging Class of Human Genetic Disorders. Annual Review of Genomics and Human Genetics, 7, 125-148. http://dx.doi.org/10.1146/annurev.genom.7.080505.115610

[6] Absalon, S., Blisnick, T., Kohl, L., Toutirais, G., Dore, G., Julkowska, D., et al. (2008) Intraflagellar Transport and Functional Analysis of Genes Required for Flagellum Formation in Trypanosomes. Molecular Biology of the Cell, 19, 929-944. http://dx.doi.org/10.1091/mbc.E07-08-0749

[7] Taschner, M., Bhogaraju, S. and Lorentzen, E. (2012) Architecture and Function of IFT Complex Proteins in Ciliogenesis. Differentiation, 83, S12-S22. http://dx.doi.org/10.1016/j.diff.2011.11.001

[8] Delaval, B., Bright, A., Lawson, N.D. and Doxsey, S. (2011) The Cilia Protein IFT88 Is Required for Spindle Orientation in Mitosis. Nature Cell Biology, 13, 461-468. http://dx.doi.org/10.1038/ncb2202

[9] Pazour, G.J., Dickert, B.L., Vucica, Y., Seeley, E.S., Rosenbaum, J.L., Witman, G.B., et al. (2000) Chlamydomonas IFT88 and Its Mouse Homologue, Polycystic Kidney Disease Gene tg737, Are Required for Assembly of Cilia and Flagella. The Journal of Cell Biology, 151, 709-718. http://dx.doi.org/10.1083/jcb.151.3.709

[10] Schrick, J.J., Onuchic, L.F., Reeders, S.T., Korenberg, J., Chen, X.N., Moyer, J.H., et al. (1995) Characterization of the Human Homologue of the Mouse Tg737 Candidate Polycystic Kidney Disease Gene. Human Molecular Genetics, 4, 559-567. http://dx.doi.org/10.1093/hmg/4.4.559

[11] You, N., Liu, W., Tang, L., Zhong, X., Ji, R., Zhang, N., et al. (2012) Tg737 Signaling Is Required for Hypoxia-Enhanced Invasion and Migration of Hepatoma Cells. Journal of Experimental Clinical Cancer Research, 31, 75. http://dx.doi.org/10.1186/1756-9966-31-75

[12] McIntyre, J.C., Davis, E.E., Joiner, A., Williams, C.L., Tsai, I.C., Jenkins, P.M., et al. (2012) Gene Therapy Rescues Cilia Defects and Restores Olfactory Function in a Mammalian Ciliopathy Model. Nature Medicine, 18, 1423-1428. http://dx.doi.org/10.1038/nm.2860

[13] Robert, A., Margall-Ducos, G., Guidotti, J.E., Bregerie, O., Celati, C., Brechot, C., et al. (2007) The Intraflagellar Transport Component IFT88/Polaris Is a Centrosomal Protein Regulating G1-S Transition in Non-Ciliated Cells. Journal of Cell Science, 120, 628-637. http://dx.doi.org/10.1242/jcs.03366

[14] Freitas, M.J., Korrodi-Gregorio, L., Morais-Santos, F., da Cruz, E.S.E. and Fardilha, M. (2014) TCTEX1D4 Interactome in Human Testis: Unraveling the Function of Dynein Light Chain in Spermatozoa. Omics, 18, 242-253. http://dx.doi.org/10.1089/omi.2013.0133

[15] Meng, Q., Lux, A., Holloschi, A., Li, J., Hughes, J.M., Foerg, T., et al. (2006) Identification of Tctex2beta, a Novel Dynein Light Chain Family Member That Interacts with Different Transforming Growth Factor-Beta Receptors. Journal of Biological Chemistry, 281, 37069-37080. http://dx.doi.org/10.1074/jbc.M608614200

[16] Dinkel, H., Michael, S., Weatheritt, R.J., Davey, N.E., Van Roey, K., Altenberg, B., et al. (2012) ELM-The Database of Eukaryotic Linear Motifs. Nucleic Acids Research, 40, D242-D251. http://dx.doi.org/10.1093/nar/gkr1064

[17] de Castro, E., Sigrist, C.J., Gattiker, A., Bulliard, V., Langendijk-Genevaux, P.S., Gasteiger, E., et al. (2006) ScanProsite: Detection of PROSITE Signature Matches and Prorule-Associated Functional and Structural Residues in Proteins. Nucleic Acids Research, 34, W362-W365. http://dx.doi.org/10.1093/nar/gkl124

[18] Letunic, I., Doerks, T. and Bork, P. (2012) SMART 7: Recent Updates to the Protein Domain Annotation Resource. Nucleic Acids Research, 40, D302-D305. http://dx.doi.org/10.1093/nar/gkr931

[19] Karpenahalli, M.R., Lupas, A.N. and Soding, J. (2007) TPRpred: A Tool for Prediction of TPR-, PPR- and SEL1-Like Repeats from Protein Sequences. BMC Bioinformatics, 8, 2. http://dx.doi.org/10.1186/1471-2105-8-2

[20] Blom, N., Sicheritz-Ponten, T., Gupta, R., Gammeltoft, S. and Brunak, S. (2004) Prediction of Post-Translational Glycosylation and Phosphorylation of Proteins from the Amino Acid Sequence. Proteomics, 4, 1633-1649. http://dx.doi.org/10.1002/pmic.200300771

[21] Blom, N., Gammeltoft, S. and Brunak, S. (1999) Sequence and structure-Based Prediction of Eukaryotic Protein Phosphorylation Sites. Journal of Molecular Biology, 294, 1351-1362. http://dx.doi.org/10.1006/jmbi.1999.3310

[22] de Castro, E., Sigrist, C.J., Gattiker, A., Bulliard, V., Langendijk-Genevaux, P.S., Gasteiger, E., et al. (2006) ScanPro- 
site: Detection of PROSITE Signature Matches and ProRule-Associated Functional and Structural Residues in Proteins. Nucleic Acids Research, 34, W362-W365. http://dx.doi.org/10.1093/nar/gkl124

[23] Petersen, T.N., Brunak, S., von Heijne, G. and Nielsen, H. (2011) SignalP 4.0: Discriminating Signal Peptides from Transmembrane Regions. Nature Methods, 8, 785-786. http://dx.doi.org/10.1038/nmeth.1701

[24] Bologna, G., Yvon, C., Duvaud, S. and Veuthey, A.L. (2004) N-Terminal Myristoylation Predictions by Ensembles of Neural Networks. Proteomics, 4, 1626-1632. http://dx.doi.org/10.1002/pmic.200300783

[25] Petersen, B., Petersen, T.N., Andersen, P., Nielsen, M. and Lundegaard, C. (2009) A Generic Method for Assignment of Reliability Scores Applied to Solvent Accessibility Predictions. BMC Structural Biology, 9, 51. http://dx.doi.org/10.1186/1472-6807-9-51

[26] del-Toro, N., Dumousseau, M., Orchard, S., Jimenez, R.C., Galeota, E., Launay, G., et al. (2013) A New Reference Implementation of the PSICQUIC Web Service. Nucleic Acids Research, 41, W601-W606. http://dx.doi.org/10.1093/nar/gkt392

[27] Hendrickx, A., Beullens, M., Ceulemans, H., Den Abt, T., Van Eynde, A., Nicolaescu, E., et al. (2009) Docking MotifGuided Mapping of the Interactome of Protein Phosphatase-1. Chemistry Biology, 16, 365-371. http://dx.doi.org/10.1016/j.chembiol.2009.02.012

[28] Reid, A.T., Redgrove, K., Aitken, R.J. and Nixon, B. (2011) Cellular Mechanisms Regulating Sperm-Zona Pellucida Interaction. Asian Journal of Andrology, 13, 88-96. http://dx.doi.org/10.1038/aja.2010.74

[29] Mannowetz, N., Kartarius, S., Wennemuth, G. and Montenarh, M. (2010) Protein Kinase CK2 and New Binding Partners during Spermatogenesis. Cellular and Molecular Life Sciences, CMLS, 67, 3905-3913. http://dx.doi.org/10.1007/s00018-010-0412-9

[30] Lamb, J.R., Tugendreich, S. and Hieter, P. (1995) Tetratrico Peptide Repeat Interactions, To TPR or Not to TPR? Trends in Biochemical Sciences, 20, 257-259. http://dx.doi.org/10.1016/S0968-0004(00)89037-4

[31] Barlow, C., Liyanage, M., Moens, P.B., Tarsounas, M., Nagashima, K., Brown, K., Rottinghaus, S., Jackson, S.P., Tagle, D., Ried, T. and Wynshaw-Boris, A. (1998) Atm Deficiency Results in Severe Meiotic Disruption as Early as Leptonema of Prophase I. Development, 125, 4007-4017.

[32] Cohen, P.T. (2002) Protein Phosphatase 1-Targeted in Many Directions. Journal of Cell Science, 115, 241-256.

[33] Yamazaki, H., Nakata, T., Okada, Y. and Hirokawa, N. (1995) KIF3A/B: A Heterodimeric Kinesin Superfamily Protein that Works as a Microtubule Plus End-Directed Motor for Membrane Organelle Transport. Journal of Cell Biology, 130, 1387-1399. http://dx.doi.org/10.1083/jcb.130.6.1387

[34] Chen, J., Yu, L., Li, D., Gao, Q., Wang, J., Huang, X., Bi, G., Wu, H. and Zhao, S.Y. (2003) Human CRYL1, a Novel Enzyme-Crystallin Overexpressed in Liver and Kidney and Downregulated in 58\% of Liver Cancer Tissues from 60 Chinese Patients, and Four New Homologs from Other Mammalians. Gene, 302, 103-113. http://dx.doi.org/10.1016/S0378-1119(02)01095-8

[35] Erlich, H.A., Valdes, A.M., Julier, C., Mirel, D. and Noble, J.A. (2009) Evidence for Association of the TCF7 Locus with Type I Diabetes. Genes and Immunity, 10, S54-S59. http://dx.doi.org/10.1038/gene.2009.92

[36] Guyot, R., Vincent, S., Bertin, J., Samarut, J. and Ravel-Chapuis, P. (2010) The Transforming Acidic Coiled Coil (TACC1) Protein Modulates the Transcriptional Activity of the Nuclear Receptors TR and RAR. BMC Molecular Biology, 11, 3. http://dx.doi.org/10.1186/1471-2199-11-3

[37] Hou, X., Mrug, M., Yoder, B.K., Lefkowitz, E.J., Kremmidiotis, G., D’Eustachio, P., Beier, D.R. and Guay-Woodford, L.M. (2002) Cystin, a Novel Cilia-Associated Protein, Is Disrupted in the cpk Mouse Model of Polycystic Kidney Disease. Journal of Clinical Investigation, 109, 533-540. http://dx.doi.org/10.1172/JCI0214099

[38] Hu, S.S., Sun, W.Y., Wei, W., Wang, D., Jin, J., Wu, J.J., Chen, J.Y., Wu, H.X. and Wang, Q.T. (2013) Involvement of the Prostaglandin E Receptor EP2 in Paeoniflorin-Induced Human Hepatoma Cell Apoptosis. Anticancer Drugs, 24, 140-149. http://dx.doi.org/10.1097/CAD.0b013e32835a4dac

[39] Murakami, H., Mizuno, T., Taniguchi, T., Fujii, M., Ishiguro, F., Fukui, T., et al. (2011) LATS2 Is a Tumor Suppressor Gene of Malignant Mesothelioma. Cancer Research, 71, 873-883. http://dx.doi.org/10.1158/0008-5472.CAN-10-2164

[40] Pontoglio, M. (2000) Hepatocyte Nuclear Factor 1, a Transcription Factor at the Crossroads of Glucose Homeostasis. Journal of the American Society of Nephrology, 11, S140-S143.

[41] To, S.Q., Takagi, K., Miki, Y., Suzuki, K., Abe, E., Yang, Y., Sasano, H., Simpson, E.R., Knower, K.C. and Clyne, C.D. (2012) Epigenetic Mechanisms Regulate the Prostaglandin E Receptor 2 in Breast Cancer. Journal of Steroid Biochemistry and Molecular Biology, 132, 331-338. http://dx.doi.org/10.1016/j.jsbmb.2012.07.007

[42] Zheng, M., Zheng, K., Zhou, J.F., Sun, H.Y., Huang, L.F. and Liu, W.L. (2012) Tcf-1 Gene Silence Suppresses Downstream Gene Expression in CD4(+) T Cells from Bone Marrow of Aplastic Anemia Patients. Annals of Hematology, 91, 353-358. http://dx.doi.org/10.1007/s00277-011-1314-0 
Scientific Research Publishing (SCIRP) is one of the largest Open Access journal publishers. It is currently publishing more than 200 open access, online, peer-reviewed journals covering a wide range of academic disciplines. SCIRP serves the worldwide academic communities and contributes to the progress and application of science with its publication.

Other selected journals from SCIRP are listed as below. Submit your manuscript to us via either submit@scirp.org or Online Submission Portal.
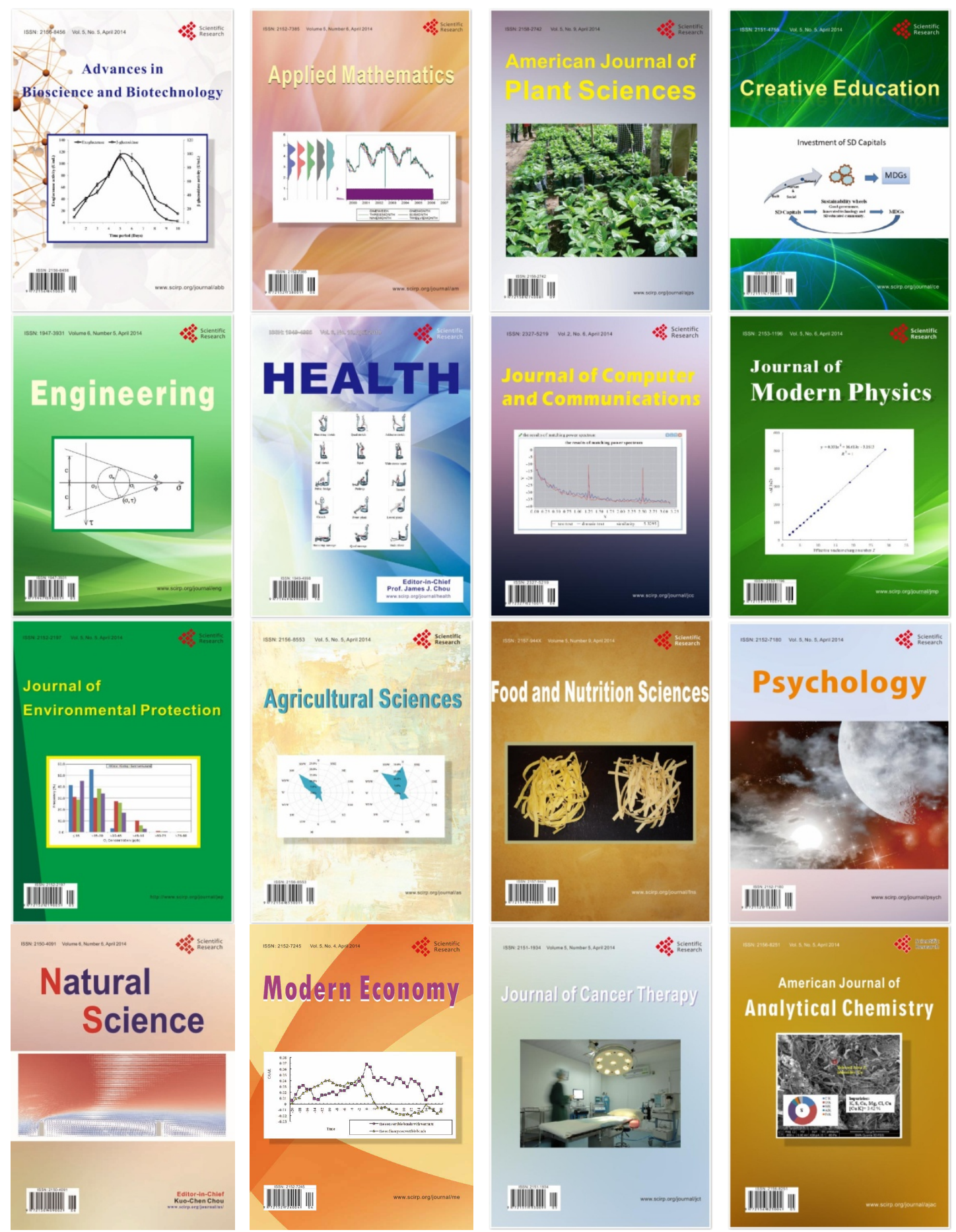